\section{La cultura de paz en el departamento del Atlántico}

The culture of peace in the Atlantico

department

Maury Almanza-Iglesia' - Raimundo Caviedes-Hoyos² - Cristobal Arteta-Ripoll³

\begin{abstract}
ACPPTAD: 23-05-16
\end{abstract}
\section{Resumen:}

Desde un enfoque interdisciplinar, el programa pretende indagar, analizar e interpretar los hechos de lo anterior y que indican, sea como hechos o como signos y símbolos, la implementación de la cultura de Paz, a partir de la voluntad o de la institucicionalidad, en las diferentes organizaciones e instituciones

se trata, de estudiar los cambios en las formas de comportamiento social, que confirman o no, la presencia

Pabras clave: cultura de paz, comportamiento social, instituciones, organizaciones, implementación.

Abstract

m an interdisciplinary perspective, the programa seeks to investigate, analyze and interpret the essentia social normes and actions From the above and that indicate the sea as facts as signs and symbols, the implementation of the Culture of Peace, based on the will of the institution, in the different organizations and public and private institutions of the Atlantic state. Finally, it is a question of studying the changes
in the forms of social behavior, which confirm or not the presence of the implementation of a culture of eace in the Region.

Keywords: peace culture, social behavior, institutions, organizations, implementation

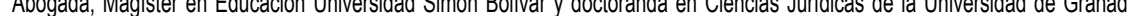
España. Invesigiadodora Junior del Grupo de linvestigación Derecchos Humanos, Cultura de Paz, Confilictos y Posconflicio.

(a)

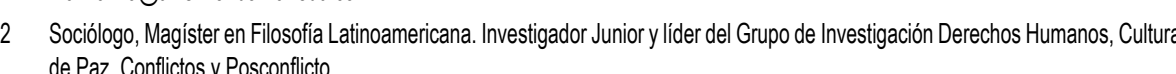
de Paz, Confilicos y Posconflicto.
cavivedes(Qunisimonobolivareduco

3 Licenciado en Ciencias Sociales y Econónicas de la Universidad del Attanitico, Magister en Educación Filissofia Latinoamericana Investigador del Grupo de Invesigación Derechos Humanos, Cultura de Paz, Confilicos y Posconfilictos.

cristobalaretel@yahoo.es
La presente ponencia tiene por finalidad analizar en un Programa de Investigación la cultura de paz en el departamento del Atlántico, su implementación y del mismo modo el comportamiento de la sociedad respecto a este para una real construcción de una cultura de paz desde la gestión pacífica de conflictos, estudiando la aplicación de esta desde las distintas instituciones y organizaciones para su mejor promoción y mejor gestión desde todos los puntos de vista sociales; para tal fin, se abordará la cultura de paz como una nueva forma de construcción social desde el ordenamiento jurídico-político colombiano teniendo en cuenta: Valores, normatividad social, costumbres y demás, hasta llegar a la real implementación de este para con la sociedad, identificando primeramente los diferentes conceptos que sobre el mismo se han construido desde los diferentes autores, las características propias que determinan la cultura de paz en nuestra actualidad, seguidamente se definirá la influencia de la cultura de paz para verificar su efectiva aplicación en el departamento del Atlántico, dentro de este se presentarán diferentes análisis de hechos que nos servirán para determinar su conclusión que busca dejar abierta la puerta a futuras investigaciones que den mayor profundidad a cada uno de los temas abordados por este artículo.

Es un hecho incontrovertible y conocido por todos, que hasta el momento ha existido en Colombia una cultura predominantemente violenta, que no significa solo un estado de guerra, sino también costumbres, valores, imaginarios, entre otros, que denotan y connotan la existencia de la violencia en el comportamiento social.

Sin embargo, no todo puede decirse que es violencia y cultura de la violencia en Colombia y específicamente en Barranquilla. Existen organizaciones consolidadas e instituciones sociales que han venido trabajando por una cultura de paz. Vale la pena señalar las organizaciones relacionadas con los derechos humanos y aquellas que abogan por una justicia social, una mayor ampliación de nuestra esquiva democracia y una participación decidida en la resolución de conflictos en forma pacífica. Aquí vale la pena destacar los esfuerzos hechos por los consultorios jurídicos de los Programas de Derecho, que han jugado un papel importante en lo relacionado con la convivencia y solución de conflictos en paz; los centros de conciliación también de los Programas de Derecho, así como los centros de conciliación privados, los jueces de paz, la unidad de víctimas, en las comunidades indígenas, el palabrero que juega 
el papel de conciliador para la solución de conflictos. Además existen otras muchas organizaciones tanto privadas como públicas y ONG, que trabajan en las comunidades campesinas y barrios populares, de las que no se conoce mucho acerca de sus esfuerzos en el trabajo y la implementación de una cultura de paz. Esas prácticas son las que se pretende sacar a la luz en la investigación para conocer su experiencia, establecer los avances y desarrollos, asî como sus alcances y cambios dentro del contexto donde vienen trabajando.

Las investigaciones derivadas de este programa es aportar un nuevo conocimiento sobre prácticas y costumbres interpretadas desde un enfoque de cultura de paz, permitirán orientar a instituciones, organizaciones públicas y privadas, asi como al ciudadano de a pie en la práctica de una cultura de paz ligada a las políticas de desarrollo sostenible que se impartan en lo local y regional.

\section{OBJETIVOS GENERALES}

Conocer las prácticas, usos, costumbres y procedimientos que favorecen la cultura de paz en las diferentes organizaciones sociales del departamento del Atlántico y examinar los efectos de su implementación en el bienestar de estas y de sus actores, para aprender de las experiencias y contribuir a situar procesos, develar obstáculos y acompañar acciones tendientes a la democratización y participación ciudadana.

\section{OBJETIVOS ESPECIFICOS E INDICADORES}

1. Reconocer experiencias significativas para una cultura de paz en las instituciones, asociaciones de vecinos, ONG y empresas privadas del departamento del Atlántico, identificando tanto los obstáculos como las habilidades, destrezas, hábitos y prácticas para superarlos.

Indicadores: Acontecimientos, episodios, hechos, historias, precedentes, ocurrencias, buenas prácticas, ejemplos de vida, entre otros

2. Recopilar y examinar documentación en instituciones, asociaciones de vecinos, ONG y empresas privadas del departamento del Atlántico, que favorezcan u obstaculicen la cultura de paz.

Indicadores: Normas, leyes, Declaraciones, manuales de convivencia, de funciones, Peis, Centros de Conciliación, estructuras organizativas, consejerías, programas, entre otros.
3. Analizar acciones de denuncia y resistencia pacífica, en el departamento del Atlántico, contra la exclusión, la discriminación, el totalitarismo, la injusticia, la inequidad, la corrupción, el armamentismo.

Indicadores: Movimientos, comunicados, informes, asambleas, llamados, graffitis, documentales, Videos (Defensoría del Pueblo, Oficina de Derechos Humanos).

4. Analizar actos públicos relacionados con la cultura de paz en el departamento del Atlántico.

Indicadores: Eventos relacionados con: Educación para la paz, fomento del diálogo, igualdad de género, cooperación internacional, desarrollo sostenible, cohesión social, participación ciudadana, derechos humanos, promoción del conocimiento libre, libertad de información, entre otros.

5. Analizar políticas públicas relacionadas con la paz.

Indicadores: Educación para la paz, fomento del diálogo, igualdad de género, cooperación internacional, desarrollo sostenible, cohesión social participación ciudadana, derechos humanos, promoción del conocimiento libre, libertad de información, entre otros.

\section{METOdOLOGía}

Como se ha señalado en reiteradas ocasiones, la paz no se logrará con solo la firma de acuerdos, sino que es necesario producir transformaciones en la sociedad tanto en el ámbito económico-social, como en la cultura, para que esta sociedad llegue a ser más tolerante y pacífica. Este papel solo podrá realizarse desde las instituciones de la sociedad civil, especialmente las escuelas, las universidades, las empresas, las ONG, la iglesia, entre otras instituciones y organizaciones. Por ello, desde el punto de vista metodológico, se trata de indagar de qué manera ellas, promueven formas que coadyuven a la paz, sea a través de medios o generando al interior de la misma organización prácticas de cultura de paz. La indagación se realizará a través de métodos cuantitativos y cualitativos. Además en cada uno de los grupos de instituciones se realizará un seminario donde cada organización fundamente, a través de ponencias, de qué manera contribuyen ellas a la generación de una cultura de paz.

Entre unos de los apartes del Programa de Investigación de la Cultura de Paz en el departamento del Atlántico, se abordará en primera instancia desde la disciplinar de la Ciencia Jurídica, con un paradigma histórico hermenéutico y el 
enfoque documental como fuente primaria por ser las estrategias de análisis los documentos (Leyes, Decretos, Sentencias de la Corte Constitucional).

En segunda instancia con el campo transdisciplinar de la Paz, Galtung (1990), que se entrelazará con otras disciplinas Sociología Jurídica, Ciencias Políticas, Historia del Derecho, Filosofía del Derecho, etc., asumiendo la complejidad como lo propone Francisco Muñoz y Beatriz Molina (2004), desde una matriz unitaria y comprensiva que incluye la paz imperfecta, conflictos, mediación, se tomará la mediación como herramientas de construcción de realidades de paz imperfecta, concepto que nos proporciona unas bases metodológicas que podrían resumirse en el reconocimiento de la dimensión positiva y creativa de los conflicto y el reconocimiento de carácter imperfecto, inacabado y procesal de la Paz, y por tanto la potenciación de la paz, mediante estrategias de regulación pacífica de los conflictos frente a las violencias. La cultura de paz y concretamente los indicadores de esta cultura de paz, tal como lo estipula la Resolución de Naciones Unidas y las medidas recomendadas para la consagración de indicadores (Almanza Iglesia M., 2014).

\section{CATEGORÍAS DE ANÁLISIS}

Formas pacíficas de resolución de conflictos

- Democracia y participación

- Derechos humanos.

Cooperación

- Equidad.

Altruismo, Inclusiones.

Trato digno

- Justicia.

- Solidaridad.

- Formas pacíficas de resistencia.

\section{ENFOQUES DE PAZ}

A raíz de las conversaciones que se llevaron a cabo en La Habana entre el Gobierno y las FARC, se suscitaron en Colombia una serie de discusiones alrededor del tema de la paz: ¿qué es? ¿cómo se llega a ella? 0 ¿como se construye? Ninguna de estas preguntas es exclusiva del conflicto y posconflicto en Colombia. También han sido formuladas en otros escenarios y países donde han tenido lugar conflictos armados entre naciones como conflictos internos. A lo largo de los estudios sobre la paz, han venido apareciendo conceptos tales como: Paz positiva, paz negativa, paz imperfecta. Igualmente se han ido desarrollando conceptos como violencia simbólica, violencia cultural, estructural, entre otros. En escenarios de conflictos armados internos y como forma de resolución de los mismos han prevalecido fundamentalmente tres enfoques: minimalista, maximalista y un enfoque intermedio. Caviedes, R. Arteta, C. y Mora, R. (2017) señalan que:

1. El enfoque minimalista reduce la consecución de la paz, a la "entrega" de armas por parte de los grupos fuera de la ley, en este caso la guerrilla, al sometimiento a la justicia para pagar penas. Así mismo se invertiría en la reconstrucción de lo que quedó destruido durante el conflicto, por ejemplo, la infraestructura eléctrica o de carreteras, las escuelas, hospitales, iglesias, entre otras. Pero no se mete con las instituciones, las costumbres, usos, y valores, que deben continuar igual.

2. El enfoque maximalista no solo considera la necesidad de acabar con las condiciones que dieron origen al conflicto sino que es necesario también generar desarrollo económico, político y social para superar las causas estructurales tales como la pobreza, la inequidad y la exclusión. Así mismo considera la necesidad de ampliar la democracia para una mayor y efectiva participación de la población en los destinos del país, y lo que es más importante, promover el cambio de usos y costumbres para la construcción de una cultura de paz en todas las relaciones sociales.

3. La postura o punto de vista intermedia, si bien plantea la necesidad de invertir en el desarrollo económico, para disminuir la pobreza, la exclusión, dar mayor cobertura a lo social, vale decir mayor empleo, salud y educación entre otros aspectos, no llega a plantearse la necesidad de construcción de un nuevo ethos, que cambie nuestros usos y costumbres y principalmente el tratamiento de los conflictos.

En el marco legal para la paz, y específicamente en Justicia Transicional, también se expresan los mismos enfoques, aunque la Fundación Ideas para 
la Paz incluye otro: la aproximación holística o integral, como lo plantea el representante de este organismo. Así tenemos de acuerdo con Palou (2013):

La aproximación maximalista: Considera que los violadores de derechos humanos -de un régimen autoritario o de un conflicto interno- se deben someter a estrictos procesos judiciales para evitar que en el futuro se produzcan nuevas violaciones. De esa manera se establece y legitima el Estado de Derecho y se fortalece la democracia. De no hacerse así, se está promoviendo la cultura de la impunidad, se erosionan las bases del Estado de Derecho y se fomenta la justicia privada.

La aproximación minimalista: Quienes sostienen este enfoque dudan que sea cierta la afirmación de que los juicios penales tienen un efecto disuasivo frente a futuras violaciones de derechos humanos. Se inclinan por las amnistías amplias pues consideran que los procesos penales incrementan la violencia y ponen en peligro los procesos de cambio, pues aumentan la resistencia de quienes se oponen a las transformaciones democráticas. Por lo demás, opinan que el maximalismo justiciero es ciego frente a las limitaciones y realidades políticas que impiden o dificultan el juzgamiento de todos los perpetradores. Los minimalistas hacen más énfasis en el polo transicional que en el de la justicia.

La aproximación moderada: Esta posición podría estar representada por las Comisiones de la Verdad, que son instrumentos equidistantes entre los procesos penales de los maximalistas y las amnistías de los minimalistas. A los primeros se los satisface con base en que estas Comisiones tienen un importante componente de rendición de cuentas (accountability) y los segundos creen que son un buen reemplazo de los procesos penales pues al tiempo que promueven el conocimiento de la verdad no generan tanta resistencia por parte de los saboteadores (...)

La aproximación holística o integral: Este enfoque se separa de los tres anteriores por considerar que aquellos reducen demasiado los mecanismos para luchar por los derechos humanos y para manejar el legado de violencia del pasado. Esta posición es promovida por el ICT) (...)

La aproximación integral u holística consiste pues en abrir el abanico de mecanismos para lograr la combinación de ellos que mejor se adecúe a las muy disimiles situaciones y contextos que deben ser enfrentados en los procesos transicionales.

\section{PAZ NEGATIVA Y PAZ POSITIVA}

Uno de los estudiosos y activistas más connotados hoy en el tema de la paz, según (Calderón, 2009) es J. Galtung, quien insiste en el proyecto de paz por medios pacíficos. Ello supone un cambio de paradigma, tema que ya fue iniciado por Gandhi en el siglo XIX. Se trata del paso de la concepción según la cual la paz únicamente se construye por medios violentos a la concepción de la consecución de la paz por medios pacíficos. Tanto Gandhi como Galtung, se basan en las siguientes premisas de acuerdo con (Calderón, 2009, p.65):

1. La violencia solo puede generar más violencia, mientras que la no violencia generará siempre no violencia.

2. La paz por medios pacíicos tiene que ser afrontada con much racionalidad y profundo respeto por el hombre y sus necesidades básicas (bienestar, libertad, identidad y sobrevivencia).

3. El proyecto de paz por medios pacíficos, pone al hombre como punto de partida, no a ideologías, credos, partidos políticos, países, etc.

De acuerdo con esta visión la consecución de la paz total pasa por tres etapas: Paz negativa, que supone el sometimiento y la guerra; la paz positiva que supone cooperación y desarrollo; y la paz cultural o cultura de paz que supone nuevas realidades, hábitos y usos.

De lo anterior se colige que la cuestión de la paz entendida en su sentido holístico sobrepasa la coyuntura de un posconflicto para transformarse en un proyecto sociopolítico, cultural y psicológico, que debe tener en cuenta las características de cada región del país.

Ubicado en la Región Caribe de Colombia, su capital es Barranquilla. A pesa de ser el departamento más pequeño de la región Caribe, es el más poblado. Tiene una extensión de $3.386 \mathrm{~km} 2$ y cuenta con 2,373,550 habitantes. Limita: Al norte con el mar Caribe; al este con el río Magdalena, desde Bocas de Ceniza 
hasta el Canal del Dique; al sur con el departamento de Bolívar; al oriente con el río Magdalena y el departamento del Magdalena. Tiene 22 municipios y un Distrito Especial, Industrial y Portuario: Barranquilla.

El departamento del Atlántico, apesar de tener una tradición y una cultura no tan proclive a la violencia política y delincuencia organizada, en las últimas tres décadas, vio amenazada la paz y la tranquilidad por el crimen en sus diferentes formas y manifestaciones.

Efectivamente, muy a pesar de que su capital, Barranquilla, se convirtió en punto de convergencia de los desplazados de muchas partes del país, la impresión que se tenía era que existía un distanciamiento del conflicto armado que desde hace años vive el Litoral Caribe, sobre todo la zona aledaña a la Sierra Nevada de Santa Marta y sus dos departamentos vecinos: el Magdalena y Bolivar.

Pero el distanciamiento solo era parcialmente, pues las guerrillas utilizaban al Departamento como corredor para desplazarse entre esos dos departamentos, además, contaban con células de formación de cuadros políticos y guerrilleros, cooptados en algunos colegios, universidades y sindicatos.

A partir de los inicios de la segunda década del presente siglo, aproximadamente desde el año 2003, el Bloque Norte de las denominadas Autodefensas Unidas de Colombia, se trazan como política incursionar en el departamento del Atlántico, bajo la dirección del jefe paramilitar Rodrigo Tovar Pupo "Jorge 40", para contrarrestar la influencia guerrillera, generar influencia política sobre gobernantes municipales, controlar las finanzas públicas en el mayor número de municipios, proclives al chantaje y a la corrupción, además de manejar a su antojo negocios ilegales como el narcotráfico. Política que no hubiese dado resultado si no hubiese tenido el consentimiento de autoridades civiles, militares, testaferros y cómplices.

Entre 2003 y 2006 el exmayor del Ejército José Pablo Díaz, asumió la responsabilidad de la dirección del Bloque Norte, asignada por Jorge 40, pero muy pronto ante su inesperada muerte asumió como sustituto el excapitán del Ejército Edgar Ignacio Fierro, alias "Don Antonio", quien cumplió con eficacia la criminal tarea encomendada.
Durante ese periodo, según informes recogidos de las entidades estatales como la Policía, el Ejército, la Vicepresidencia de la República, la Fiscalía y la Defensoría del Pueblo, y, según cifras publicadas el 11 de octubre de 2011 por (Acnur, 2011) solo en el año 2004 fueron asesinadas en el departamento del Atlántico setecientas treinta (730) personas, en el 2005 seiscientas treinta y siete (637) y en el 2006 seiscientas nueve (609). En Barranquilla, entre el 2003 y el 2006 el número de asesinatos ascendió a mil seiscientas sesenta y cuatro (1664) personas y en el mismo periodo, en Soledad quinientos setenta y dos (572) y en Malambo ciento dieciséis (116).

Todos esos asesinatos respondieron, en algunos casos, a la política de limpieza de guerrilleros, a la mal denominada limpieza social, a la lucha por el control del tráfico de drogas y a la retaliación contra quienes no respondieran al chantaje, boleteo y extorsión de las Autodefensas. Igualmente la ofensiva paramilitar en la región se extendió hacia los defensores de derechos humanos, académicos, estudiantes, sindicalistas y políticos de izquierda, a quienes amenazaron, desplazaron, intimidaron y asesinaron.

Con la captura del paramilitar Don Antonio en marzo del 2006, fue posible establecer, gracias a la información suministrada por su computador personal, la manera como funcionaba el Bloque Norte en la Región, cómo accionaban su política, el detalle de sus acciones delictivas, el listado de supuestos guerrilleros y colaboradores de la guerrilla en la academia, organizaciones sindicales y políticas. En posteriores declaraciones ante la Fiscalía señaló los nexos con los organismos de seguridad del Estado, como la Policía, el Ejército, el Das, quienes proporcionaban información para identificar víctimas, amenazarlas, desplazarlas o asesinarlas. Según informe de la Defensoría del Pueblo entre 2005 y 2006, fueron amenazadas 890 personas, entre sindicalistas, estudiantes y docentes, lo cual demuestra que ese contubernio propició el desarrollo de la guerra política sucia contra líderes sindicales y políticos, en el departamento del Atlántico y su capital.

En las audiencias públicas el grupo paramilitar comandado por Don Antonio se responsabilizó, según (Fiscalía General de la Nación, 2016) un documento de la Unidad de Justicia y Paz, de la muerte selectiva de 245 personas y del desplazamiento forzado de más de 500 . Como se observa, sin mediar combate alguno y constituyéndose esos crímenes de guerra en grave violación de los derechos humanos y en delitos de lesa humanidad. 
Igualmente, en versión libre, Miguel Villarreal Archila, alias "Salomón", subintendente retirado de la Policía, quien fue designado por Don Antonio para dirigir una nueva estructura criminal denominada Comisión al Mar, admitió la complicidad de los organismos del Estado con sus fechorías criminales. Según esa declaración, por los puertos del Atlántico, entre el año 2003 y 2006, se enviaron al exterior más de 1000 toneladas de coca, con un impuesto por cada kilo que oscilaba entre 50 y 75 dólares.

Empresarios, tenderos e industriales, estuvieron comprometidos con esas actividades ilícitas, toda vez, que cedieron a la extorsión, cancelando con puntualidad la vacuna de la que fueron víctimas por años. Solo por concepto de la vacuna a tenderos se calcula, según información proporcionada por UNDECO (Artuz, 2012), que los paramilitares recibieron una suma superior a los 3.000 millones de pesos mensuales. Una cantidad superior debieron recibir, de parte de empresarios e industriales para no atentar contra sus vidas y negocios. Hasta penetraron la dirección de entidades como la Cooperativa de productos de Leche-Coolechera-, para controlarla con varias finalidades, entre ellas, lavar el dinero que ganaban por concepto de impuestos al narcotráfico y utilizar sus finanzas y sus medios logisticos para delinquir. Otra fuente de financiación importante fue el control de alcaldías y entidades públicas, como ocurrió, con suma gravedad, con la Alcaldía de Soledad.

Según datos entregados por la Defensoría del Pueblo, solo en el año 2003, fueron amenazados 63 sindicalistas pertenecientes a la denominada Central Unitaria de Trabajadores (CUT) y otras organizaciones sindicales, entre ellas, la Federación Colombiana de Educadores - Fecode. Tal vez los crímenes más sonados fueron los del educador de la USB, Alfredo Correa De Andreis, los profesores Lizandro Vargas y Alfredo Castro Haider, de la UA, el líder estudiantil de la UA Luis Mesa y el abogado y reconocido líder sindical y político Miguel Antonio Espinosa.

Organismos como la Fiscalía, la Comisión Nacional de Reparación y Reconciliación, la Procuraduría y la Defensoría del Pueblo, han señalado que los daños causados por el Frente José Pablo Díaz a las comunidades del Atlántico desde el año 1999 hasta el 2006, "generaron un impacto psicológico que exige una reparación colectiva y un acompañamiento de las instituciones para superar las huellas de la violencia paramilitar en la región". Lo que implica una adecuada política educativa y psicológica, un programa de liderazgo comunitario, una estrategia de promoción de la cultura de la legalidad, el control a los recursos públicos y el diálogo para la protección ciudadan

\section{REFERENCIAS BIBLIOGRÁFICAS}

Acnur (2011). Diagnóstico del departamento del Atlántico. Acnur. Bogotá: http://www. acnur.org/t3/uploads/media/COI_2163.pdf

Almanza Iglesia, M. (2014). La cultura de paz en el ordenamiento jurídico y político de Colombia: 2008-2012. En Cultura de paz y derechos humanos. Una mirada sociojurídica (pp.123-155). Barranquilla: Universidad Simón Bolivar.

Arendt H. (1993) La condición humana. Barcelona: Paidós.

Artuz, A. (29 de 11 de 2012). Tenderos en Barranquilla lanzan S.O.S. El Tiempo.

Baigorri, A. (1994). El gran miedo Burgués. (L'opinion et la multitude). Recuperado el 07 de octubre de 2015, de http://www.insumisos.com/bibliotecanew/El\%20miedo\%20 burgues.pd

Calderón, P. (2009). Teoría de conflictos de Johan Galtung. Paz y conflictos, (2), 60-81

Cavides, R. A., Arteta, C. y Mora, R. (2017). Posconflicto y Cultura de Paz en Colombia. En América Latina: Entre revoluciones y la Cultura de la Paz (p.27). Barranquilla

Córdoba, P. (23 de Julio de 2008). Palabras en el lanzamiento del libro. La subversión en Colombia. El cambio social en la historia del sociólogo Orlando Fals Borda. Recuperado el 04 de octubre de 2015, de Tiempo de mujeres. Palabras de co/2008/07/palabras-de-la-senadora-piedad-crdoba htm tocraciablogsotcom.

Fiscalía General de la Nación. (2016). Documentación: hechos Ley 975 de 2005. RAL-2016-MAY-31pdf

Freud, S. (1973). Más allá del principio de placer. En S. Freud, Obras completas T. III .

Harris, M. (s.f.). Antropología cultural. Recuperado el 15 de 10 de 2015, de http:// www.miuasinaloa.org/wp-content/uploads/2015/07/ Harris-Marvin-Antropouploads/2015/07/Haris-Manin-Antropologia-culturatpdf

Hobbes, T. (1979). Leviatán. Madrid: Nacional.

INMLCF (2014). Boletín estadístico 2014. Recuperado el 13 de octubre de 2015, de Instituto Nacional de Medicina Legal y Ciencias Forences: http://www.medicinalegal.gov.co/documents/10180/560640/11+ noviembre.pdf/353688a2-ca84-4809-

INMLCF (Julio de 2015). Forensis 2014 Datos para la vida. Datos para la vida, 16(1) Medi, Bogota, Colombia. Instituto Nacional

Kropotkin, P. (2005). El apoyo mutuo, un factor de evolución. Santiago de Chile: Instituto

Lombroso, C. (1902). El delito. Causas y remedios. En pdf. Madrid: Librería General de

Lorenz, K. (1971). Sobre la agresión. El pretendido mal. México: Siglo XXI.

Mead, M. (1975). La antropología y el mundo contemporáneo. Buenos Aires: Siglo Veinte. 
Morales, J. (2015). ¿Qué es el posconflicto? Colombia después de la guerra. Bogotá: B Colombia S.A.

Naciones Unidas (Octubre de 1999). Declaración y Programa de Acción sobre una Cultura de Paz. Recuperado el 18 de 10 de 2015, de Naciones Unidas. Asamblea General: http://www3.unesco.org/iycp/kits/sp_res243.pdf

Pacheco, R. J., Rueda, R. S. \& Amado V., C. (Noviembre de 2013). Conducta prosocial: Una alternativa a las conductas agresivas. Recuperado el 15 de 10 de 2015, de http:// investigiumire.iucesmag.edu.co/ojs/index.php/investigiumire/article/view/36: http://investigiumire.iucesmag.edu.co/ojs/index.php/investigiumire/article/ view $/ 36$

Palou, J. C. (22 de mayo de 2013). Marco legal para la paz, opción sensata y viable. Fundación Ideas para la Paz. En: http://www.ideaspaz.org/publications/posts/536

Rousseau, J. J. (2007). El contrato social. Madrid: Austral.

Rousseau, J. J. (1972). El orígen de la desigualdad entre los hombres. México: Grijalbo.

Sartre, J. P. (1985). El existencialismo es un humanismo. Barcelona: Orbis S.A.

Serbin, A. (2012). ¿Por qué la prevención de conflictos y la construcción de paz en América Latina y el Caribe? Pensamiento propio. Publicación trilingue de Ciencias Sociales de América Latina y el Caribe, (36/37), 11-61.

UNESCO (s.f). Proyecto transdisciplinario "Hacia una cultura de paz". Recuperado el 19 de 10 de 2015, de http://www.unesco.org/cpp/sp/proyectos/cppinfo.htm: http:// www.unesco.org/cpp/sp/proyectos/cppinfo.htm 